\title{
Uplift and Exhumation of the Russell Fiord and Boundary blocks along the northern Fairweather Transform Fault, Alaska
}

\author{
Anna Schartman', E. Enkelmann², J.I. Garver ${ }^{3}$, and Cameron M. Davidson ${ }^{4}$,
}

${ }^{1}$ University of Cincinnati, Department of Geology, 500 Geology Physics Building, Cincinnati, $\mathrm{OH}$ 45221-0013, USA

${ }^{2}$ University of Calgary, Department of Geoscience, 2500 University Drive N.W., Calgary, Alberta, T2N 1N4, Canada

${ }^{3}$ Union College, Department of Geology, 807 Union Street, Schenectady, NY 12308-2311, USA

${ }^{4}$ Carleton College, Department of Geology, One North College Street, Northfield, MN 55057, USA
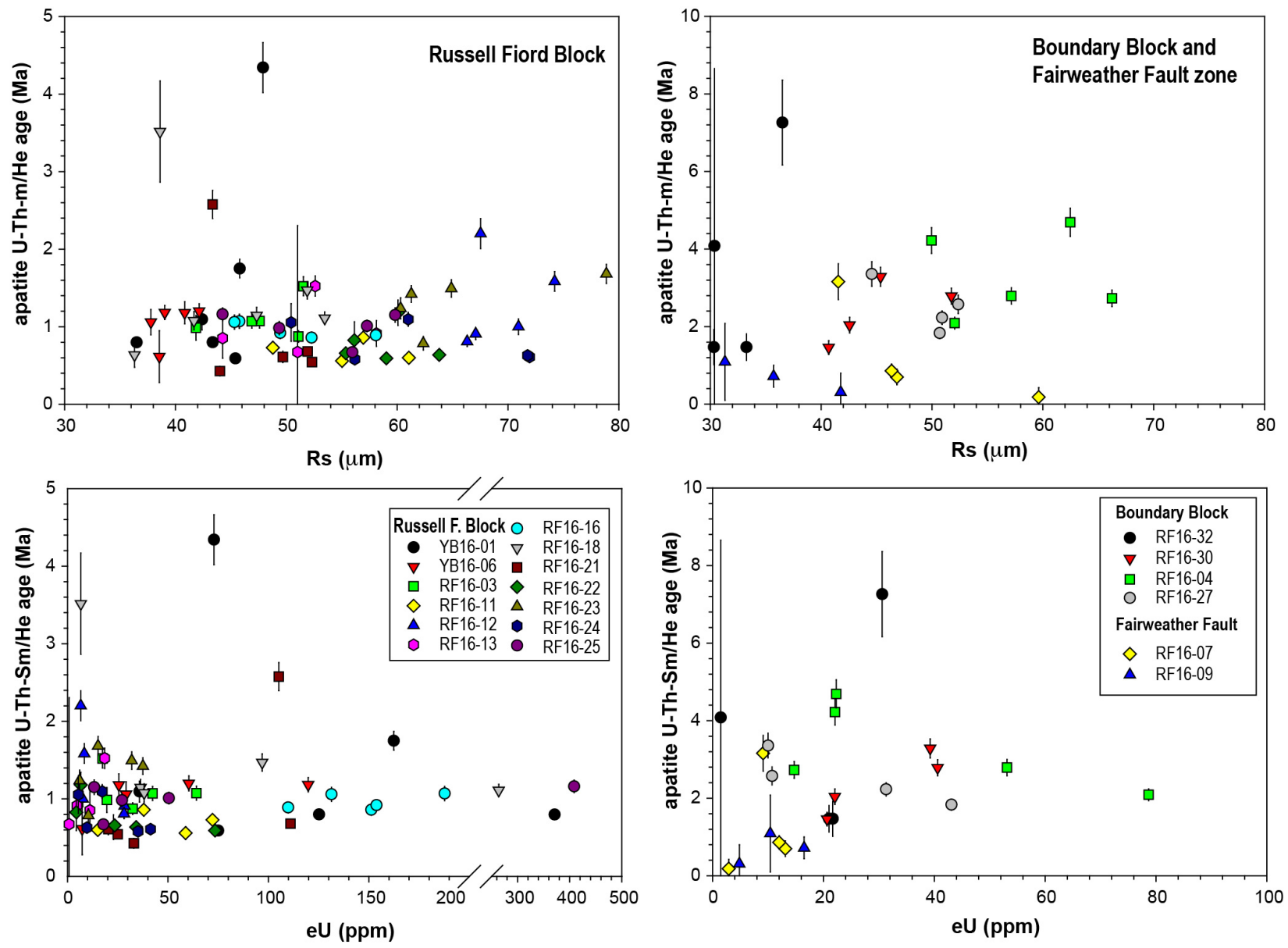

Figure S1. Apatite (U-Th-Sm)/He data plotted against the spherical radius (Rs) of the apatite grain (top) and plotted against the effective uranium concentration $(\mathrm{eU})$ of the apatite grains (bottom). Samples from the Russell Fiord block (left) are plotted separately from samples collected on the Boundary block and Fairweather Fault zone (right). 

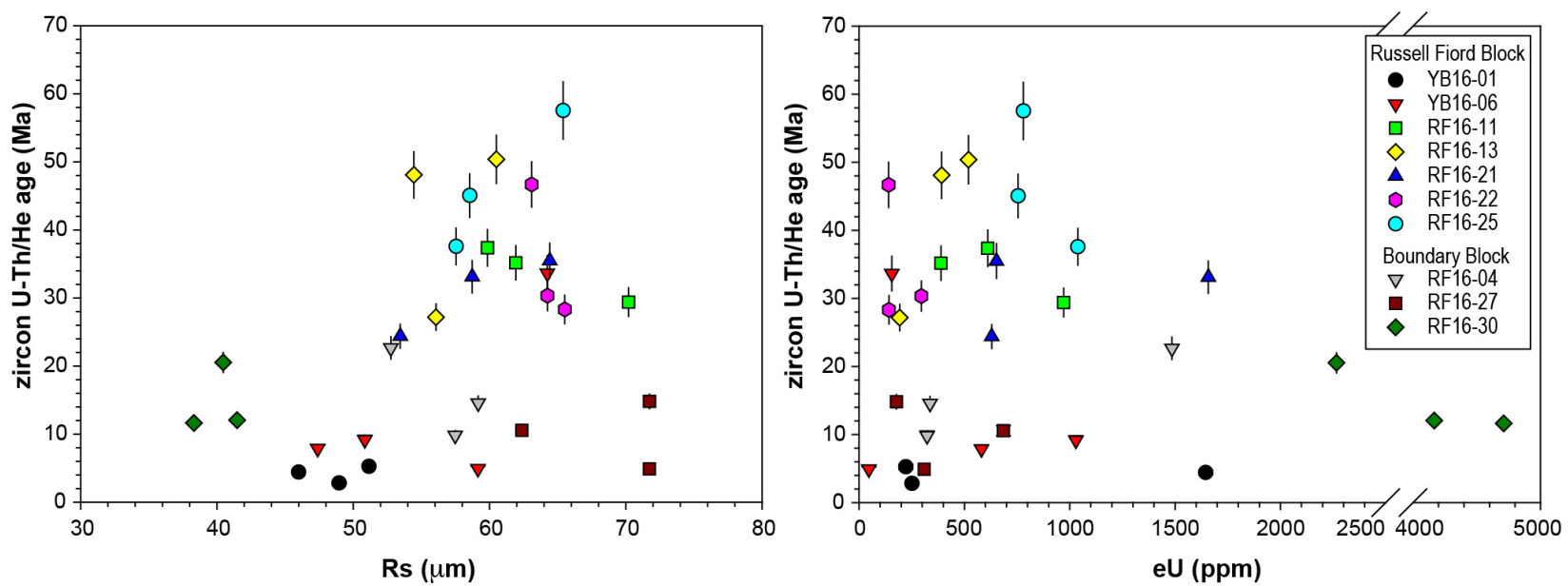

Figure S2. Zircon (U-Th)/He data plotted against the spherical radius (Rs) of the zircon grain (left) and plotted against the effective uranium concentration (eU) of the zircon grain (right).

Table S1: Parameters used for HeFTy modeling

\begin{tabular}{|c|c|c|c|c|c|c|}
\hline $\begin{array}{c}\text { Sample } \\
\text { ID }\end{array}$ & Grain IDs & $\begin{array}{c}\text { Grain age } \\
\text { Error }\end{array}$ & $\begin{array}{l}\text { Acceptable } \\
\text { fit solutions }\end{array}$ & $\begin{array}{c}\text { Good } \\
\text { Fit solutions } \\
\end{array}$ & Constraint 1 & Constraint 2 \\
\hline RF16-11 & $\mathrm{Zr} 1 / 2 / 3$ Ap $1 / 2 / 3 / 4$ & all $25 \%$ & 3479 & 1463 & $22-50 \mathrm{Ma} 70-220^{\circ} \mathrm{C}$ & $0.3-3 \mathrm{Ma} 50-120^{\circ} \mathrm{C}$ \\
\hline RF16-13 & $\operatorname{Zr} 1 / 2 / 3$ Ap $1 / 2 / 3 / 5$ & $\begin{array}{c}30 \% \text { Ap } 3 \text { all } \\
\text { others } 25 \%\end{array}$ & 4285 & 256 & $22-70 \mathrm{Ma} 80-220^{\circ} \mathrm{C}$ & $0.3-3 \mathrm{Ma} 50-120^{\circ} \mathrm{C}$ \\
\hline RF16-21 & $\operatorname{Zr~} 1 / 2 / 3 / \operatorname{Ap} 2 / 3 / 1 / 5$ & all $25 \%$ & 2500 & 1220 & $18-46 \mathrm{Ma} 80-220^{\circ} \mathrm{C}$ & $0.3-3 \mathrm{Ma} 50-120^{\circ} \mathrm{C}$ \\
\hline RF16-22 & $\mathrm{Zr} 1 / 2 / 3 / \mathrm{Ap} 1 / 2 / 4 / 5$ & all $25 \%$ & 2170 & 357 & $22-60 \mathrm{Ma} 80-200^{\circ} \mathrm{C}$ & $0.3-2.5 \mathrm{Ma} 50-120^{\circ} \mathrm{C}$ \\
\hline RF16-25 & $\mathrm{Zr} 1 / 2 / 3$ Ap $5 / 4 / 1 / 3$ & all $25 \%$ & 4060 & 1316 & $27-65 \mathrm{Ma} 80-220^{\circ} \mathrm{C}$ & $0.3-3 \mathrm{Ma} 50-120^{\circ} \mathrm{C}$ \\
\hline YA53 & $\mathrm{Zr} 1 / 2 / 3 \mathrm{Ap} 1 / 2 / 4 / 5$ & all $15 \%$ & 460 & 66 & $14-54 \mathrm{Ma} 80-220^{\circ} \mathrm{C}$ & $0.5-2 \mathrm{Ma} 50-120^{\circ} \mathrm{C}$ \\
\hline YA47 & Ap $1 / 3 / 4 / 5 \mathrm{Zr} 1 / 2$ & all $35 \%$ & 3942 & 43 & $4-35 \mathrm{Ma} \quad 80-220^{\circ} \mathrm{C}$ & $0.5-2.5 \mathrm{Ma} 50-120^{\circ} \mathrm{C}$ \\
\hline YA52 & $\mathrm{Zr} 1 / 2$ Ap $1 / 2 / 3$ & all $20 \%$ & 5384 & 806 & $1-6 \mathrm{Ma} \quad 80-220^{\circ} \mathrm{C}$ & $0.2-2 \mathrm{Ma} 50-120^{\circ} \mathrm{C}$ \\
\hline YB16-01 & $\mathrm{Zr} 1 / 2 / 3$ Ap $1 / 4 / 5 / 6$ & all $25 \%$ & 937 & 23 & $2-20 \mathrm{Ma} \quad 80-200^{\circ} \mathrm{C}$ & $0.1-3 \mathrm{Ma} 50-120^{\circ} \mathrm{C}$ \\
\hline YB16-06 & $\mathrm{Z} \mathrm{r} 2 / 3 / 4 \mathrm{Ap} 2 / 4 / 5 / 1$ & all $20 \%$ & 1784 & 18 & $3-28 \mathrm{Ma} \quad 80-220^{\circ} \mathrm{C}$ & $0.1-3$ Ma $50-120^{\circ} \mathrm{C}$ \\
\hline RF16-04 & $\mathrm{Zr} 1 / 2 / 3$ Ap 5/2/3/1 & $25 \% \mathrm{Ap} 20 \% \mathrm{Zr}$ & 976 & 5 & $6-32 \mathrm{Ma} \quad 80-220^{\circ} \mathrm{C}$ & $1-6 \mathrm{Ma} \quad 50-120^{\circ} \mathrm{C}$ \\
\hline RF16-27 & $\mathrm{Zr} 1 / 2 / 3$ Ap $1 / 2 / 3 / 5$ & $\begin{array}{c}35 \% \operatorname{Zr} 1 / 2 / 3, \\
\text { others } 25 \%\end{array}$ & 6907 & 46 & $3-26 \mathrm{Ma} \quad 80-220^{\circ} \mathrm{C}$ & $0.5-4 \mathrm{Ma} 50-120^{\circ} \mathrm{C}$ \\
\hline RF16-30 & $\operatorname{Zr} 1-3$ Ap $2 / 5 / 3 / 1$ & $\begin{array}{c}30 \% \mathrm{Zr} 1 / 2 / 3 \\
\text { others } 25 \%\end{array}$ & 4424 & 79 & $0.5-5 \mathrm{Ma} \quad 50-120^{\circ} \mathrm{C}$ & $8.5-27 \mathrm{Ma} 80-220^{\circ} \mathrm{C}$ \\
\hline YA54 & $\operatorname{Zr} 1 / 2 / 3$ Ap $1 / 2 / 3 / 4$ & $\begin{array}{c}\text { all } \mathrm{Zr} 25 \% \text { all } \mathrm{Ap} \\
30 \%\end{array}$ & 4979 & 294 & $3-22 \mathrm{Ma} \quad 80-220^{\circ} \mathrm{C}$ & $0.5-3 \mathrm{Ma} 50-120^{\circ} \mathrm{C}$ \\
\hline $\begin{array}{l}\text { YAKB58 } \\
\text { /RF16-09 }\end{array}$ & $\mathrm{Z} 1 / 2 / 3$ Ap $2 / 4 / 5$ & $\begin{array}{c}\text { all } \mathrm{Zr} 20 \% \text { all } \mathrm{Ap} \\
30 \%\end{array}$ & 113 & 0 & $1.2-4 \mathrm{Ma} 80-220^{\circ} \mathrm{C}$ & $0.2-0.6 \mathrm{Ma} 50-120^{\circ} \mathrm{C}$ \\
\hline RF16-07 & Ap 5/6/7 & all $35 \%$ & 580 & 0 & & $2-0.1 \mathrm{Ma} 50-100^{\circ} \mathrm{C}$ \\
\hline RF16-09 & Ap 2/4/ 5 & all $30 \%$ & 535 & 0 & & $0.1-2$ Ma $50-100^{\circ} \mathrm{C}$ \\
\hline
\end{tabular}

Note: For each sample the model precision was set to "Best", 50,000 simulations were conducted, the diffusion model of Flowers et al. (2009) and Guenthner et al. (2013) were used for apatite and zircon, respectively. 
Schartman, A., Enkelmann, E., Garver, J.I., and Davidson, C.M., 2019, Uplift and exhumation of the Russell Fiord and Boundary blocks along the northern Fairweather transform fault, Alaska: Lithosphere, https://doi.org/10.1130/L1011.1. GSA Data Repository Item 2019033

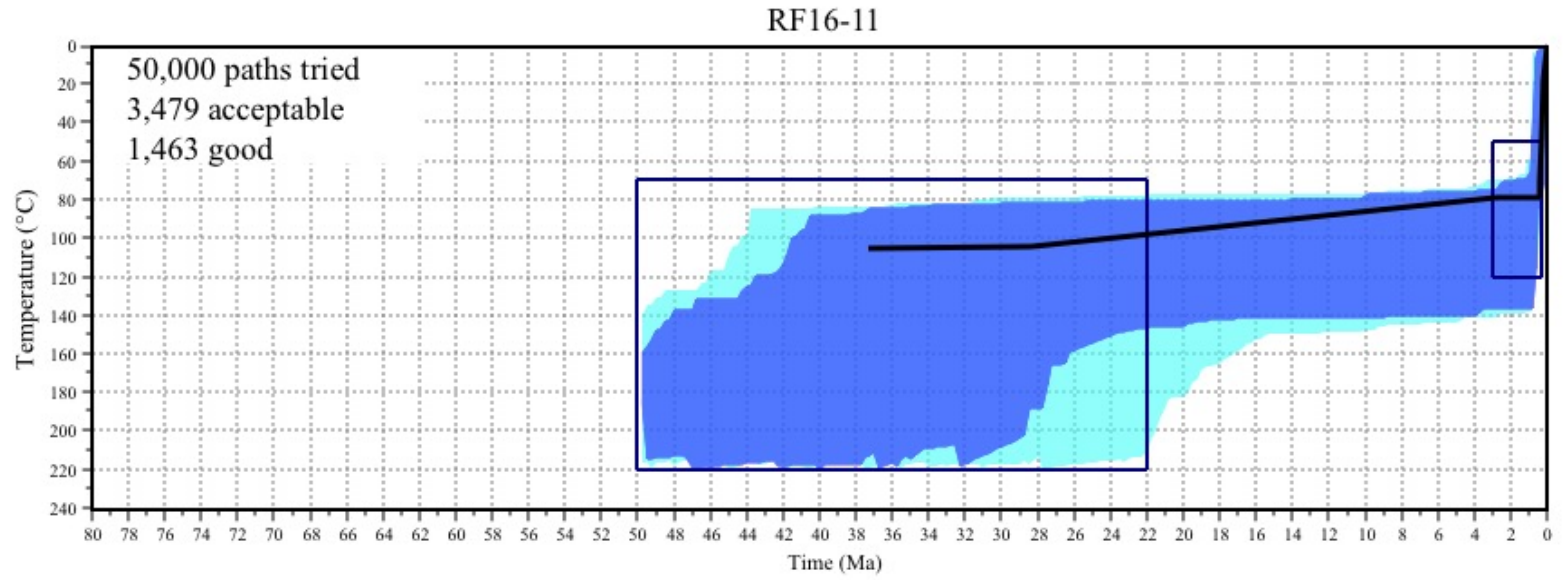

RF16-13

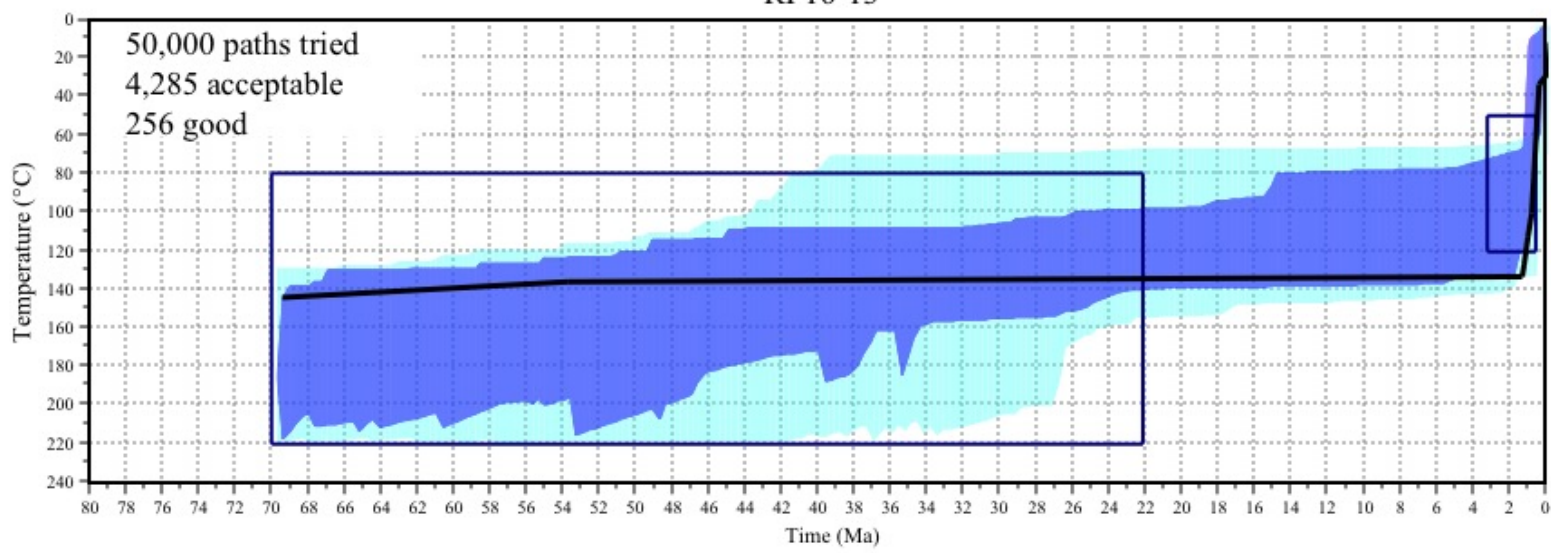

RF16-21

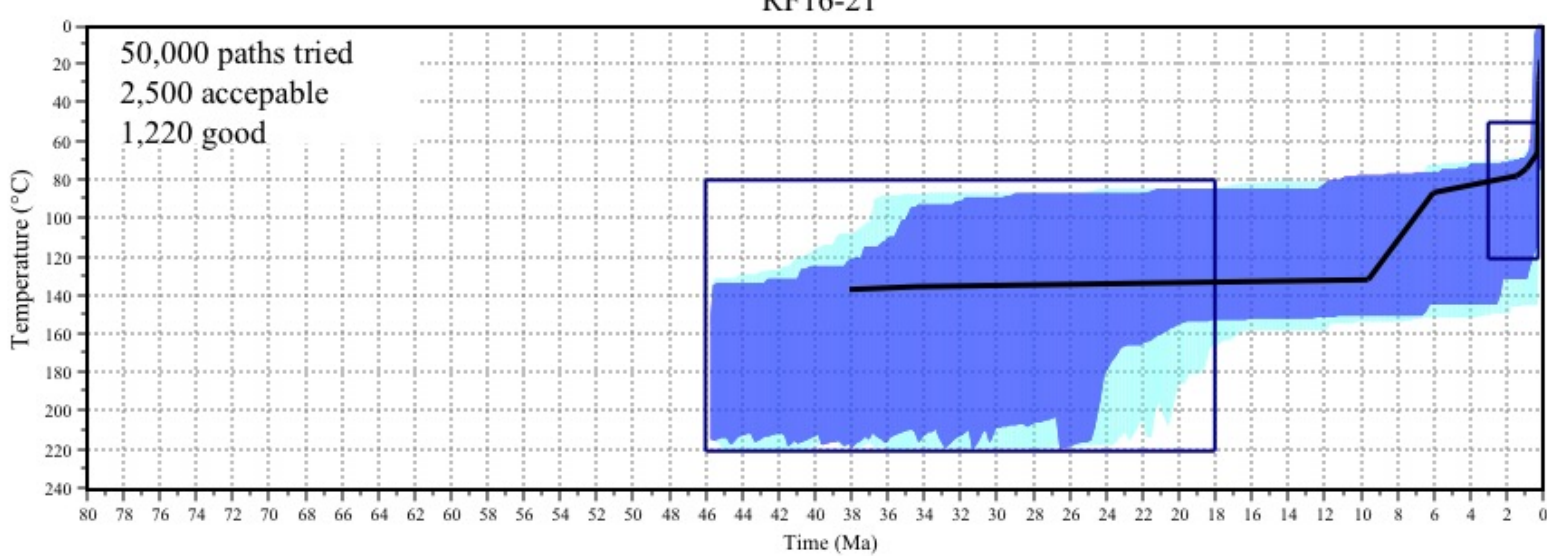


Schartman, A., Enkelmann, E., Garver, J.I., and Davidson, C.M., 2019, Uplift and exhumation of the Russell Fiord and Boundary blocks along the northern Fairweather transform fault, Alaska: Lithosphere, https://doi.org/10.1130/L1011.1.
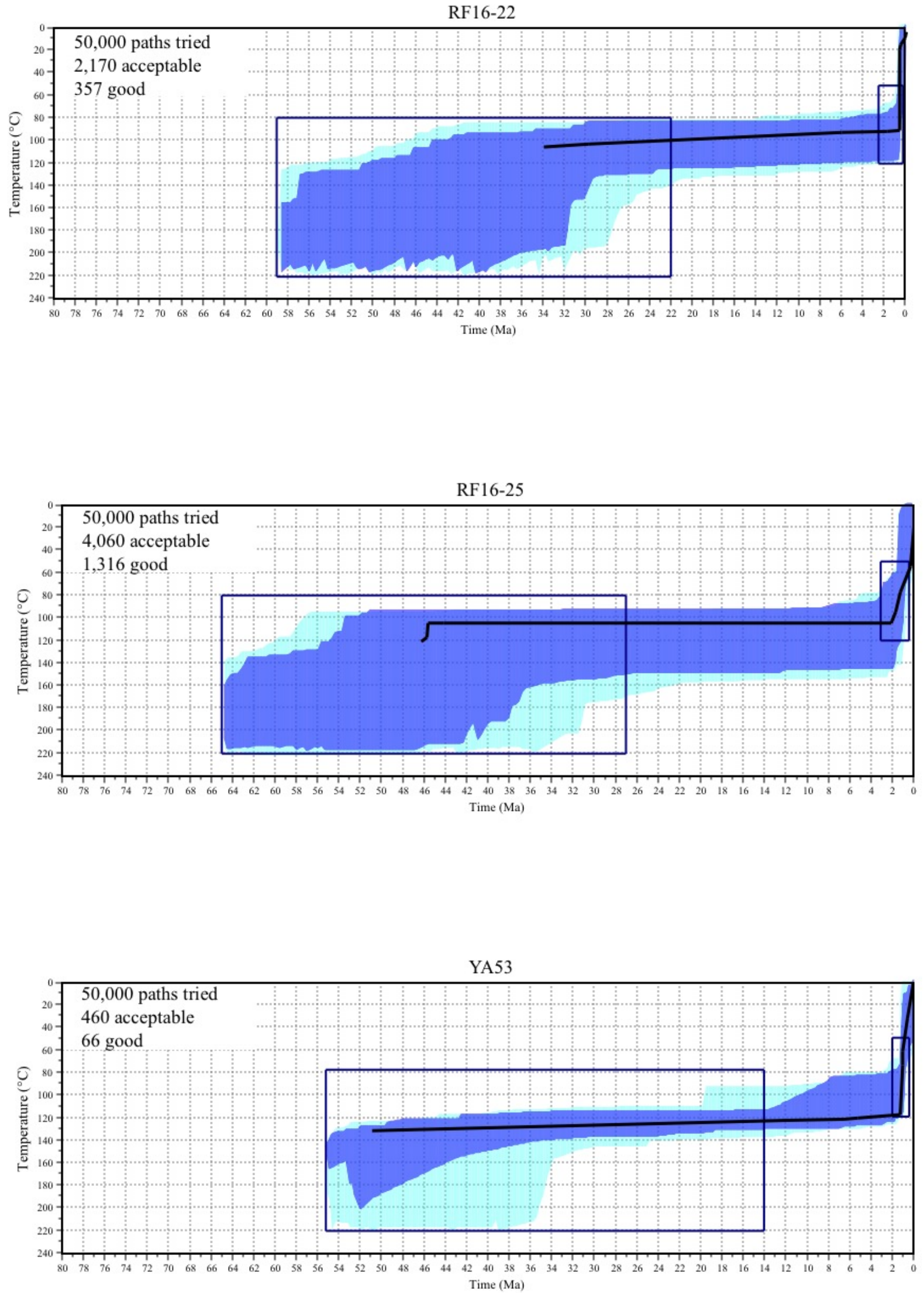
Schartman, A., Enkelmann, E., Garver, J.I., and Davidson, C.M., 2019, Uplift and exhumation of the Russell Fiord and Boundary blocks along the northern Fairweather transform fault, Alaska: Lithosphere, https://doi.org/10.1130/L1011.1.

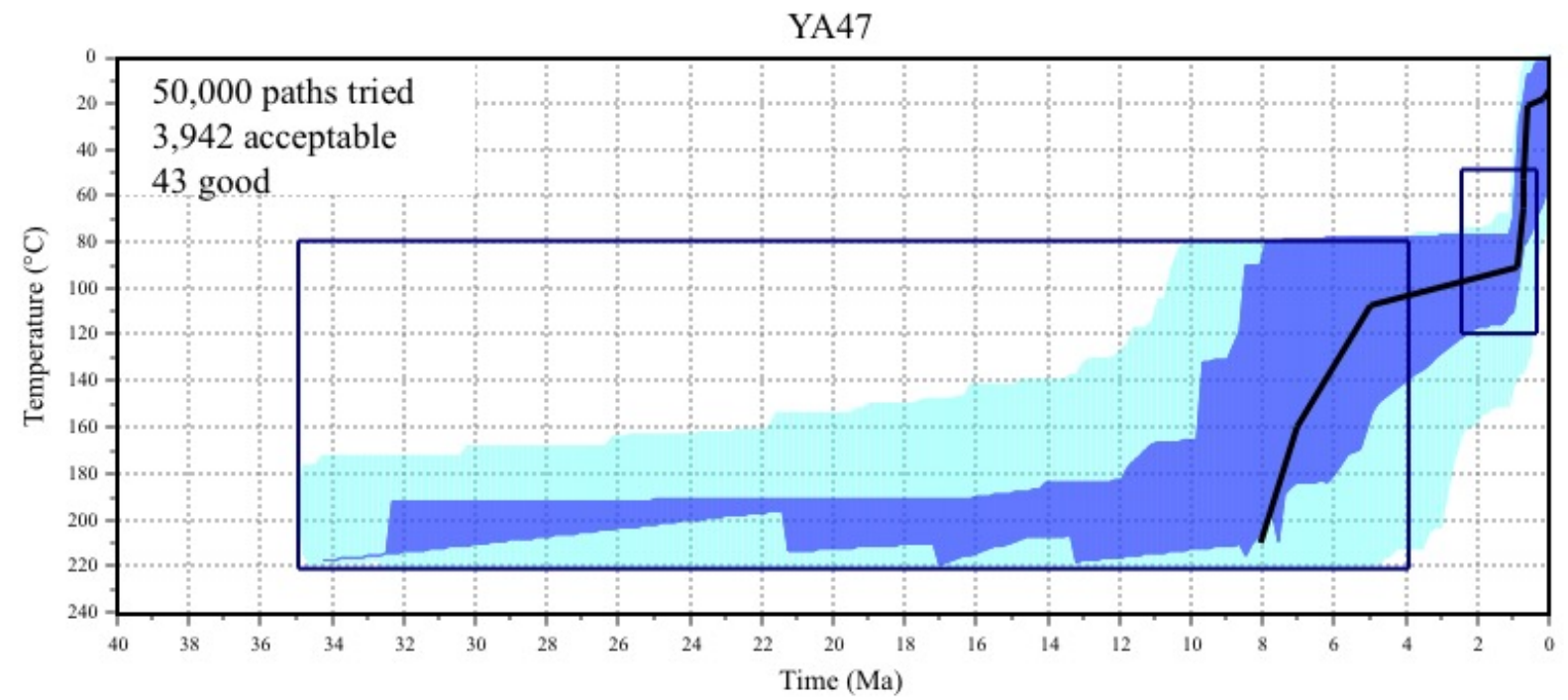

YA52
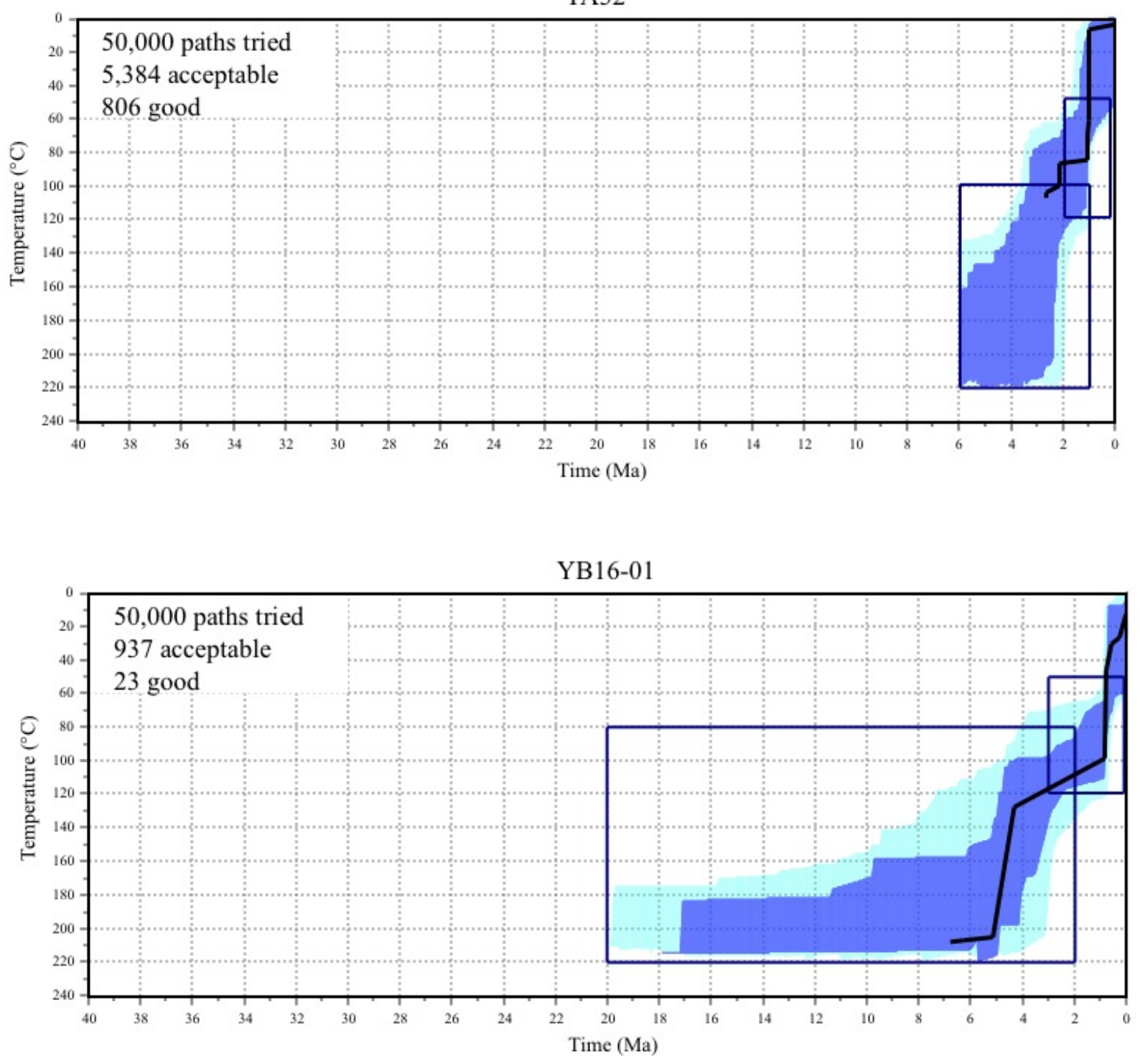

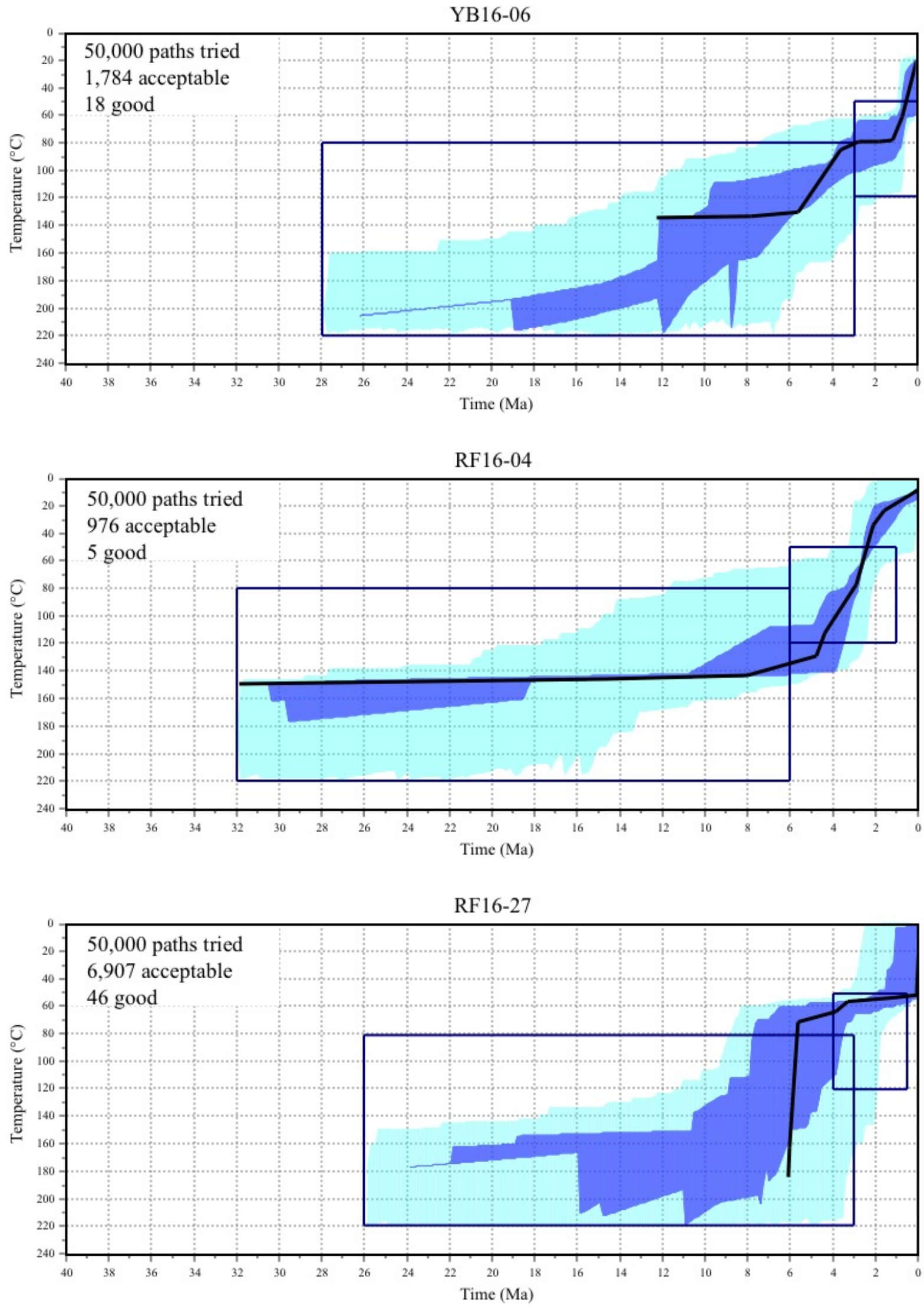
Schartman, A., Enkelmann, E., Garver, J.I., and Davidson, C.M., 2019, Uplift and exhumation of the Russell Fiord and Boundary blocks along the northern Fairweather transform fault, Alaska: Lithosphere, https://doi.org/10.1130/L1011.1.
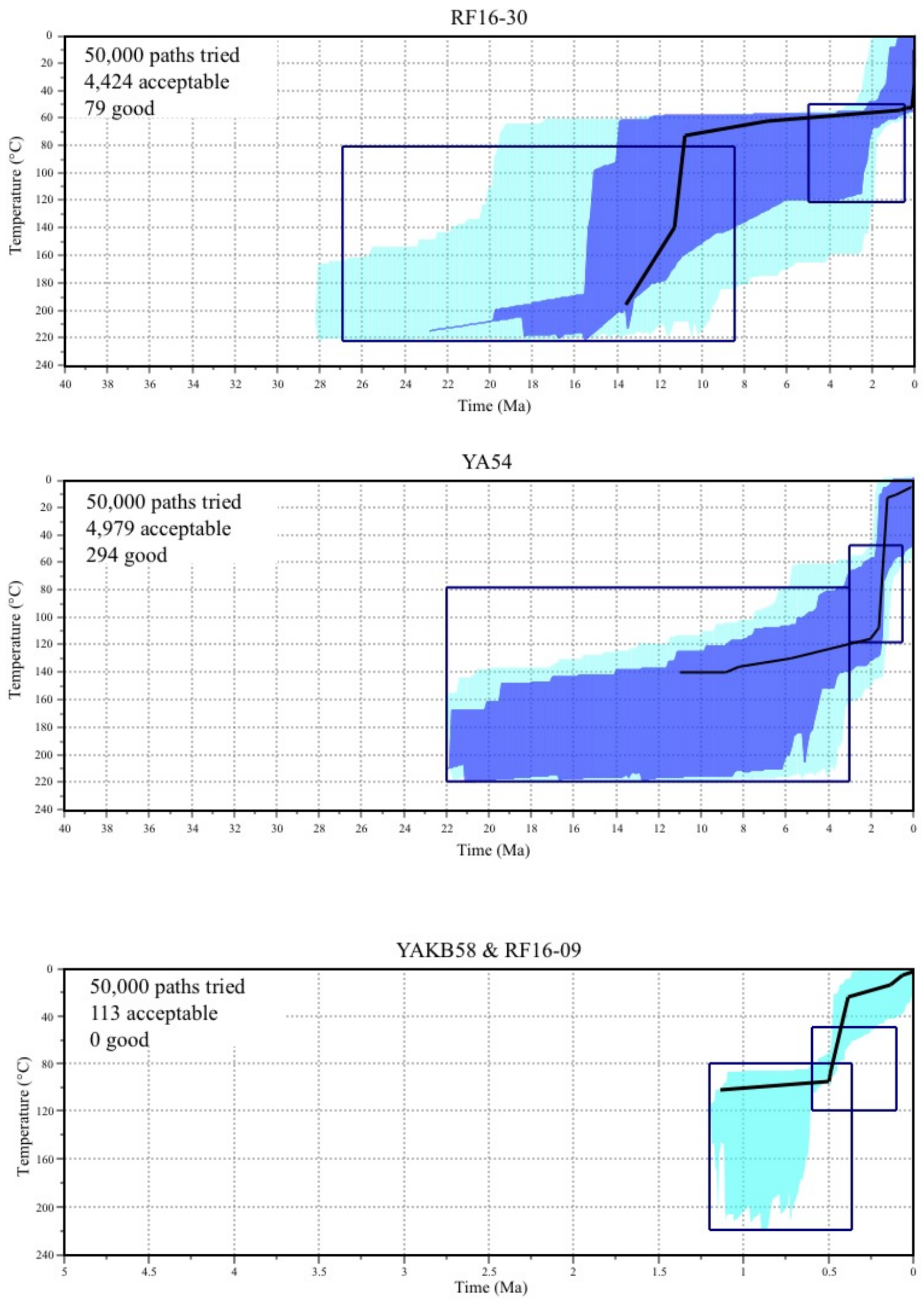

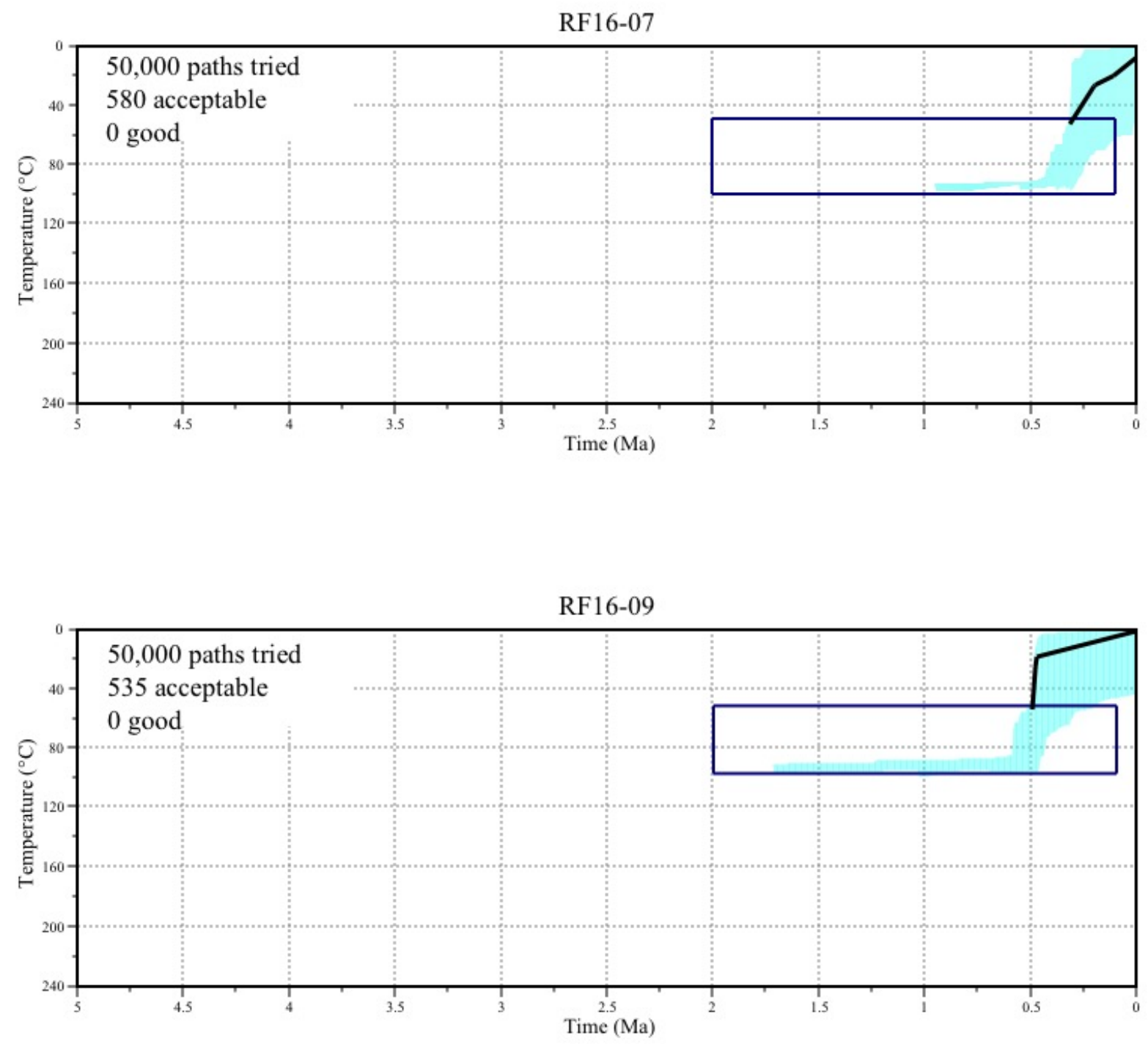

Figure S3. Time-temperature model results for individual samples. Each model has one end constraint set at $0-10^{\circ} \mathrm{C}$ at $0 \mathrm{Ma}$. Other individual constraints are depicted by the blue rectangles and listed in Table S1. Shaded light blue region indicates the envelope of acceptable solutions and dark blue the envelope of good fit solutions. The solid black lines are the best fit model for each model run consisting of 50,000 paths tried. Models generated with HeFTy (Ketcham, 2009).

\section{References:}

Flowers, R.M., Ketcham, R.A., Shuster, D.L., and Farley K.A., 2009, Apatite (U-Th)/He thermochronometry using a radiation damage accumulation and annealing model: Geochimica et Cosmochimica Acta, v. 73, no. 8, p. 2347-2365, doi:10.1016/j.gca.2009.01.015. 
Guenthner, W.R., Reiner, P.W., Ketcham, R.A., Nasdala, L., and Giester, G., 2013, Helium diffusion in natural zircon: Radiation damage, anisotropy, and the interpretation of zircon (UTh)/He thermochronology: American Journal of Science, v. 313, p. 145-198, doi: 10.2475/03.2013.01.

Ketcham, R.A., 2005, Forward and inverse modeling of low-temperature thermochronometry data: Reviews in Mineralogy and Geochemistry, v. 58, p. 275-314, doi: 10.2138/rmg.2005.58.11. 